\title{
Real Time Implementation of Face Recognition based Automatic Institutional Attendance System
}

\author{
MD. SALAH UDDIN YUSUF, AZMOL AHMED FUAD \\ Department of Electrical and Electronic Engineering \\ Khulna University of Engineering \& Technology, Khulna-9203 \\ BANGLADESH
}

\begin{abstract}
This research work addresses the issue of incorporating an automatic attendance system to the frame of an institution using face detection and recognition techniques. The proposed system aims at reducing computational time with available hardware to yield more efficient results. The proposed model utilizes Histogram Oriented Gradients and facial encodings derived from facial landmarks. It also addresses the problems related to accuracy of facial recognition and the resource requirement for quick, real-time facial recognition by applying multi-processing. The improvement in performance in terms of accuracy across two different methods, and the improvement in terms of time requirement for the same method using different strategies have also been documented for demonstration. The designed system demonstrates the effectiveness of task parallelization with a minimum amount of hardware desiderata. The system has been designed to an optimum self-sustaining ecosystem which can efficiently operate on its own accord and compute comprehensible feedback without the requirement of any third-party human interference. A Graphical User Interface has been incorporated into the system for maximum user comprehensibility.
\end{abstract}

Key-Words: - Face detection, face recognition, automation, HAAR features, LBPH, HOG, face encoding, multi-processing, attendance recording.

Received: January 24, 2021. Revised: June 14, 2021. Accepted: June 28, 2021. Published: July 5, 2021.

\section{Introduction}

Attendance recording is a must for any organization aiming to evaluate its members performance based on attendance in part. Automatic attendance recording that is accurate and requires less time and resources is hence a burning need for enormous organizations and establishments these days.

Facial Recognition process can be summarized as the process that takes a still digital image or still frame of a video derived from a video source and compares it to facial data or features of the existing facial images for the purpose of verification or identification of an individual(s). This is achieved by comparing selected facial features from given image with faces within a database. The process can be compared to other biometrics like fingerprint or eye iris recognition systems due to having typical usage like access control in security systems [1].

Its contactless and non-invasive nature makes it preferable, even though it lags behind iris and fingerprint recognition in accuracy [2]. This groundbreaking yet controversial technology has many other usages such as advanced humancomputer interaction, video surveillance, indexing of images automatically, and video database etc.
[3]. The whole process is shown in the block diagram in Figure 1 to clarify the sequence of events.

\subsection{Literature Review}

A plethora of work has been done in recent times to implement facial recognition for the purpose of recording attendance. The efficacy and accuracy of the system depends on how well it is designed or trained.

In 2000, face detection became popular when Paul Viola and Michael Jones invented an algorithm that could detect faces on even cheap cameras, known as Viola-Jones algorithm [4]. E.T. Chin et al. [5], A. Raghuwanshi et al. [6], N. D. Veer et al. [7], B. Surekha et al. [8], A. Patil et al. [9] used ViolaJones algorithm for the face detection purpose in their attendance system design. Another way to detect faces from a given image is to use HAAR

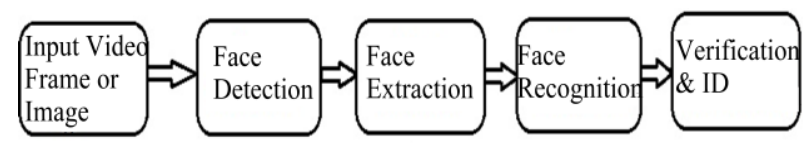

Fig. 1: Systematic flow of generic face recognition. 
features which is based on both Viola-Jones algorithm and Haar wavelet technique. B. Surekha et al. [8], A. Patil et al. [9], M. Fuzail et al. [10] specifically used Haar classifier in their face detection part. Convolutional Neural Network (CNN) and Principal Component Analysis (PCA) are also being used in face detection. CNN is a facet of deep learning that works by employing small neural networks with shared weights. The outputs are then down sampled and maxpooled to reduce in number and run through a final neural network to determine whether or not it's a match [11]. K. Rohini et al. [12] chose CNN. A. Jha [13] tested the system using PCA for the face detection part and implemented with color based technique. N. K. Balcoh et al. [14] used enhancements on the captured images and then performed face detection using Viola-Jones algorithm and face recognition using Eigenfaces. Shehu et al. [15] utilizes a single camera to capture the image and then perform face detection. D. Sunaryono et al. [16] approached attendance system based on a two-factor style authentication, using QR code and facial recognition based cross checking idea. Viola- Jones algorithm is used to perform face detection and simple classifiers like logistic regression (LR), Linear Discriminant Analysis (LDA) and k-Nearest Neighbors (k-NN).

A. Waingakar et al. [17] used a combination of systems that applies facial recognition method created by A. Geitgey [18]. The system performs facial detection and data collection by using Histogram Oriented Gradients (HOG) [19] to detect the face first. Then using facial landmark extraction proposed by Kazemi et al. [20] to detect facial features like eye, nose position etc. and use rotation to center them. Embedded faces are used to both store and compare for recognition purposes in the future. Wang et al. [21] have used R-CNN and SeetaFace [22] for face detection and recognition respectively, by taking a single frame every minute. SeetaFace is a $\mathrm{C}++$ based face recognition engine (open source) that runs on CPU without third party dependencies, developed by Visual Information Processing and Learning (VIPL) group, Institute of Computing Technology, Chinese Academy of Sciences.

\subsection{Problem Identification}

The reasons shown in the systems described here paint the picture of why using facial recognition is a good choice for larger organizations, ensuring ample accuracy. But the systems implemented here, solve the various problems of the recognition process by approaching them from different angles.
But these approaches fail to mention crucial parts like time and computational resources. Computation is required to perform comparison of the acquired image with the stored image database. But the time requirement in this process and number of computational resources required is not mentioned that clearly in these works. For commercial level, it is imperative that the system is efficient in time and computational resources without sacrificing accuracy. These systems, mostly designed for educational institutions show only until the marking stage, in rare cases notification and no further than that.

This research work, represents an Automatic Attendance System (AAS) for educational institutes, starting from the attendance recording to generating admit cards before examination and informing relevant personnel. It also focuses on the time requirement and computational resource requirement portion of the work, by showcasing them in a graphical fashion.

The rest of the paper is organized as follows. Section 2 describes the methodology proposed in this system design alongside their working principles. Results and discussion are analyzed in section 3 with graphical representations for clarification. Section 4 shows the software application of the system (GUI). Finally, section 5 concludes the research work.

\section{Proposed Methodology}

\subsection{Video Capturing or Image Acquisition}

The system currently tested with employs a HD camera as the acquisition medium for images, at the front center of the classroom. The camera may take images or record videos and store it on the drive or it can perform live detection and recognition. The system starts the camera on the user request (in this case course teacher), who logs into the system with password on the user interface of the system, also typing in the details like course number. Since the amount of test subjects is not large in this demonstration, the room segment that is required to be recorded need not be large either.

The system starts taking videos (up to 4 videos) at random intervals of 8-12 minutes in a 50-minute class. Thus, the whole time period of the class is covered with relatively small data processing. The system takes each frame and records the attendance of all the students that are recognized in that frame. Figure 2 shows the complete block diagram of the proposed automatic attendance system. 


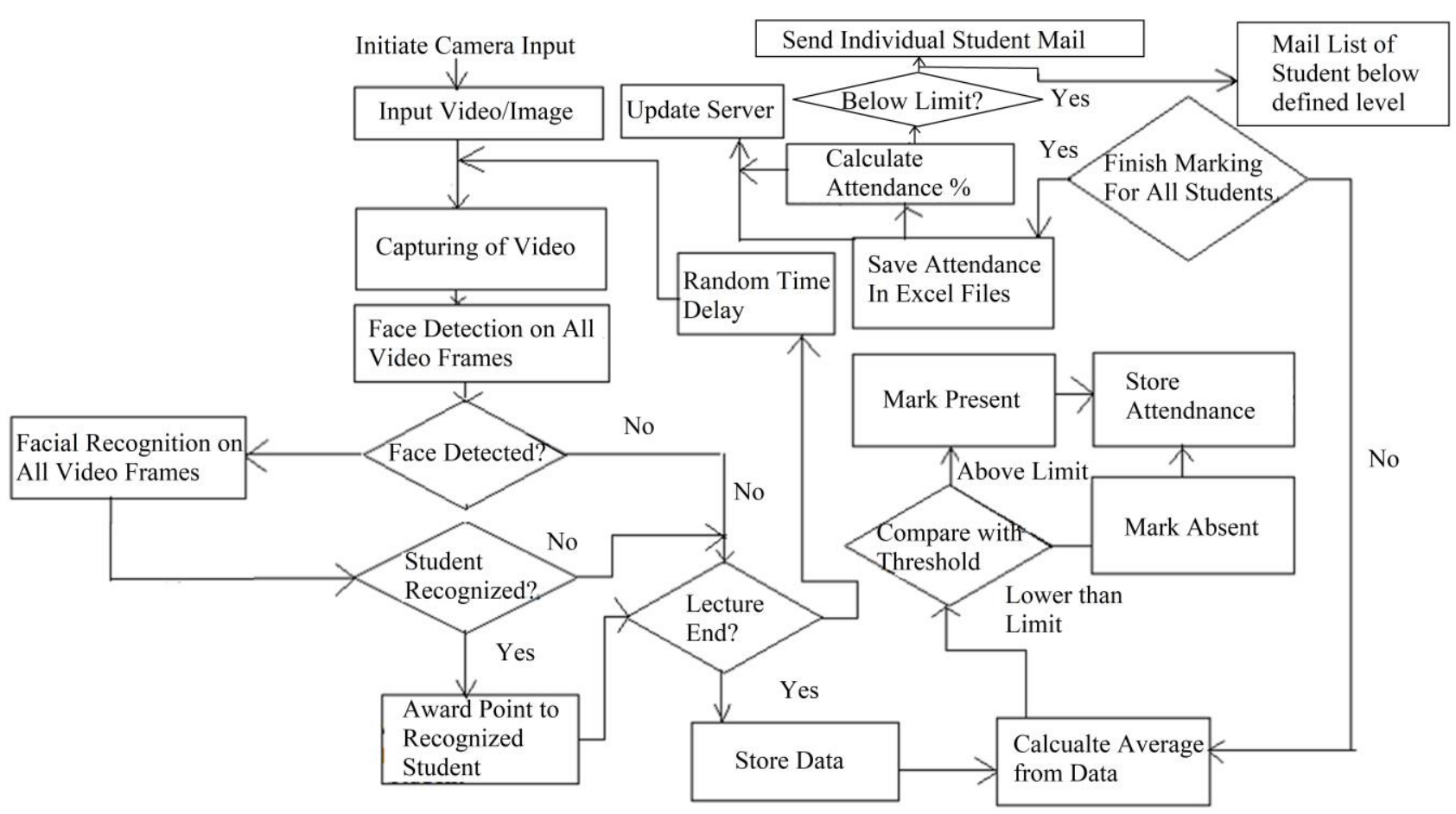

Fig. 2: Proposed automatic face recognition-based attendance system.

\subsection{Face Detection}

The model training and the recognition process are similar in working principle with an additional step for recognition process for the system. Firstly, when the system is employed with an image (or a frame) it applies Histogram Oriented Gradients (HOG) to it. HOG uses gradient arrows based on the increase of darkness direction in an image by dividing the image in small squares of $16 \times 16$ pixels. From these squares, the strongest arrow directions are used to convert the image, which is later compared to the HOG face pattern image for face detection. Figure 3 shows conversion of an input image using HOG and comparison with HOG face pattern image for face detection.

After the face is detected in an image, 68 facial landmarks [12] are found in that portion(s) of the image after the facial portions are centered enough.

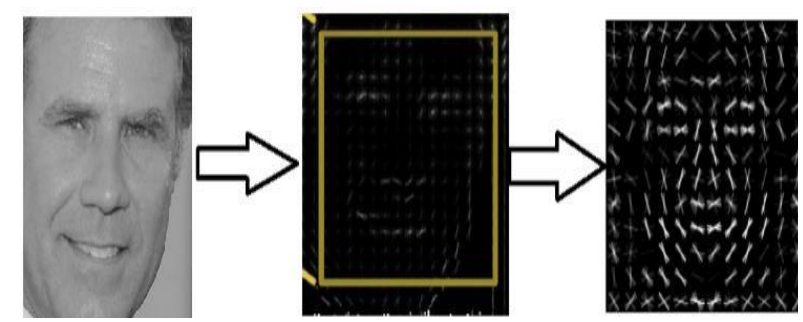

Fig. 3: Face detection using HOG features.
Centering eliminates positioning related issues during recognition and training step. The facial landmark estimation used here is a system proposed by Kazemi et al. [12]. Using this system 68 specific points landmarks are found. Figure 4 shows the facial landmarks extracted using this system that will be located during the facial database storing and recognition process. Figure 5 shows how the landmarks are extracted from an image after a face has been detected and affine transformation for the centering of the landmarks.

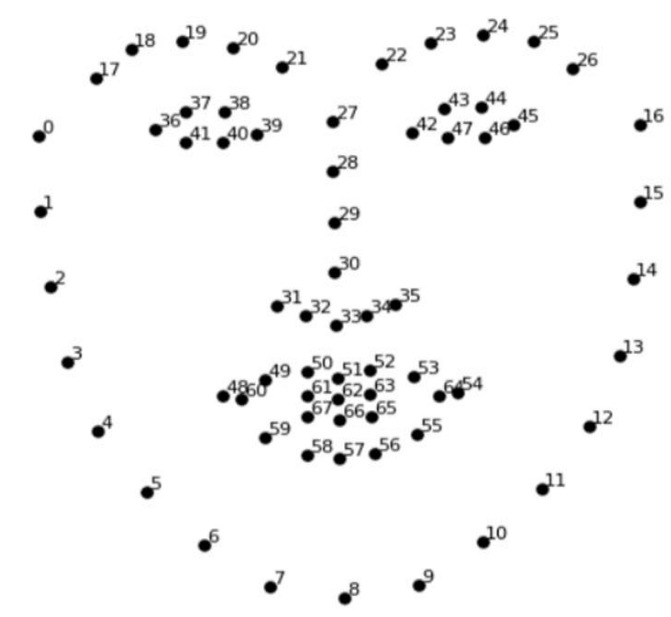

Fig. 4: Extracted 68 facial landmarks. 


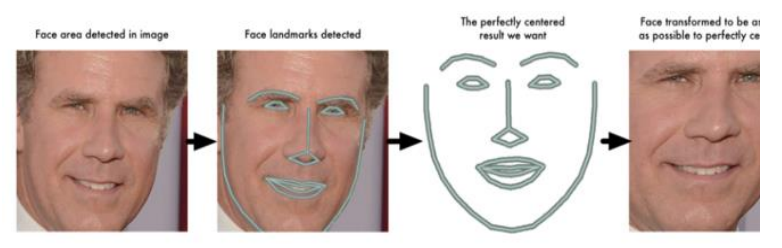

Fig. 5: Extracted landmarks and their centering.

\subsection{Face Recognition}

After facial landmarks have been detected, the system is trained to perform 128 measurements of the faces. The training is done in such a process that the same person gives almost same measurement values for every one of their images called embedding. It reduces complicated raw data of a picture into a list of computer-generated numbers. The approach used here was originally proposed by Schroff et al. [23]. The system is named Facenet and uses 128 byte per face and has a record-breaking accuracy of $99.63 \%$ on the benchmark Labeled Faces in the Wild (LFW).

For creating the embedding's and detecting the facial features, trained dlib models by King [24] are used. The model has an accuracy of $99.38 \%$ on the popular benchmark LFW and was created using a ResNet network with 29 convoluted layers. These embeddings are stored in the database and every time when an image is supplied for the recognition step, these are compared with the embedding's extracted form that image.

\subsection{Multiprocessing}

Figure 6 shows the multiprocessing techniques of frames. Multiprocessing is applied to increase the total number of frames processed in a given time and reducing the amount of total time requirement for a set of frames. This allows the computer to utilize all of its logical cores to the fullest for a short period of time to deliver the outputs faster. Frames are processed alternately to reduce the total time even more in case of pre-recorded footage. The "concurrent.futures" library of Python is used here, which returns objects after processing them in the order they were supplied. In case of a live footage, all frames are processed due to the limitations of the "multiprocessing" library, which is used in this case as it can return results while processing other frames. The frames are decoded before-hand on separate thread to reduce delay caused by the blocking operation of decoding them.

It is also worth noting that parallelization can also be done using GPU cores but they have limitations. Table 1 portrays the methods of multi-processing ways with their advantages and disadvantages.

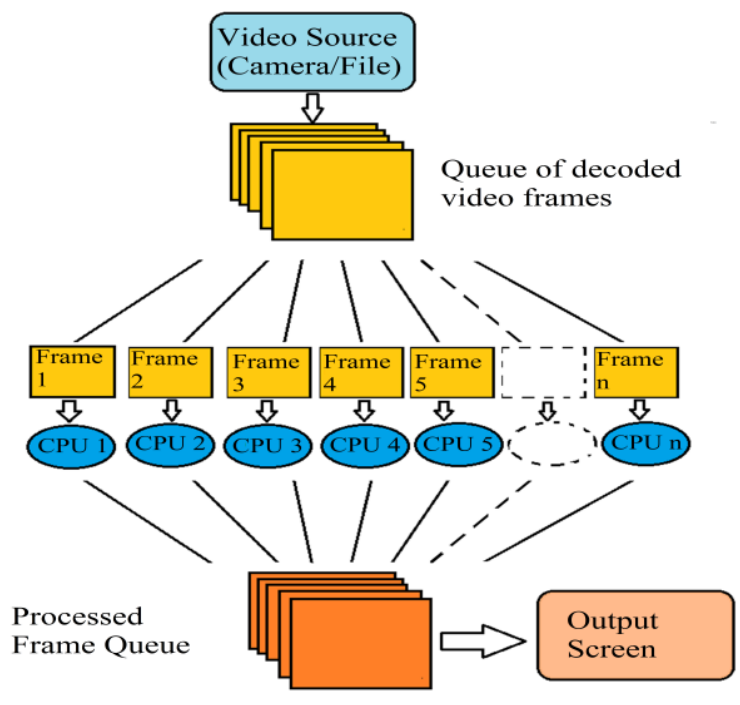

Fig. 6: Multiprocessing of frames.

Table1. Frame Multiprocessing with CPU and GPU.

\begin{tabular}{|l|l|l|}
\hline Feature & CPU & GPU \\
\hline Cost & Cheaper & Costlier \\
\hline Core Numbers & Less & More \\
\hline Workload & Various & $\begin{array}{l}\text { Graphics } \\
\text { based }\end{array}$ \\
\hline $\begin{array}{l}\text { Efficiency in Frame } \\
\text { Processing }\end{array}$ & Less & More \\
\hline $\begin{array}{l}\text { Ease of } \\
\text { Implementation }\end{array}$ & All CPU's & $\begin{array}{l}\text { Selected GPU } \\
\text { based } \\
\text { Systems }\end{array}$ \\
\hline $\begin{array}{l}\text { Availability in } \\
\text { Systems }\end{array}$ & More & Less \\
\hline $\begin{array}{l}\text { Support for Multi- } \\
\text { processing in Python }\end{array}$ & All CPU's & $\begin{array}{l}\text { Only CUDA } \\
\text { based GPU's }\end{array}$ \\
\hline
\end{tabular}

Hence CPU method has been used and recommended for this system implementation.

\subsection{Attendance Recording}

After processing and face recognition the attendance is recorded against roll number. Since multiple frames are used to take attendance multiple times, average is calculated in terms of frame numbers and number of times video captured or images supplied. Compared to a threshold value, the average determines whether or not the student would be considered present or absent during the class.

The attendance is recorded in an excel sheet specific to that course and course teacher with date stamp. This excel sheet is later processed for the final marking based on the final sheet and from that sheet email is sent automatically depending on the attendance percentage. The same final sheet is also used to generate "Admit Cards" before examination 
for the students automatically. The following section shows the results acquired form the deployed system and comparison results from different scenarios.

\section{Result and Discussion}

The results acquired can be evaluated based on comparison-based matrices and observation-based matrices. Figure 7 shows the graphical comparison between the processing time with and without parallelization. The comparison is made based on the following matrices, namely,

(i) Observed Accuracy: The measure of the accuracy between training data and result data,

(ii) Training data Requirement: The amount of data required to train the Haar based and the dlib based model respectively,

(iii) Trained Model Size: Implying the size of the trained model files between the two systems, and

(iv) Time Requirement: Pertaining to the time required by the dlib based system with and without parallelization.

The time requirement-based performance comparison also has been evaluated by testing the same system on different hardware configurations as (i) an Asus K550JX laptop running Intel core i5 $4200 \mathrm{H}$ with 2 cores 4 threads, (ii) an Asus X509FL laptop running an Intel core i5 $8265 \mathrm{U}$ with 4 cores 8 threads, and (iii) a desktop running an AMD FX8120 processor with 8 cores 8 threads. All the mentioned system had similar specifications and memory with 8 GB. Due to multiprocessing, the time requirement shows the linear decrement with the increase in logical core numbers ( 2 core system lagging behind the others). The time improvement can also be measured from the number of total frames processed. It is worth mentioning, second time improvement has been measured in the final method with multi-processing and alternate frame processing over normal processing time.

Figure 8 shows the distribution of the workload equally among all the threads or logical cores on a 4 core 8 thread machine, via the "Task Manager" of the Windows operating system. The linear decrement of time with respect to increasing number of logical cores on a machine and using alternate processing is shown in the Figure 9. The bar graphs are clustered depending on the number of frames processed and then divided depending on how they were processed (with all frames and alternate frames). The division is also based on the type of system being used. Linear decrease can be seen for both the number of frames processed in the group and the number of logical CPU core numbers. For example, the first group of 482 frames shows a processing time of 938.3 seconds for a 4-core machine with all frames processed, 528.2 seconds for every other frame processed, 479.4 seconds for 8 core machines with all frames and 252.57 seconds for half the number of frames.

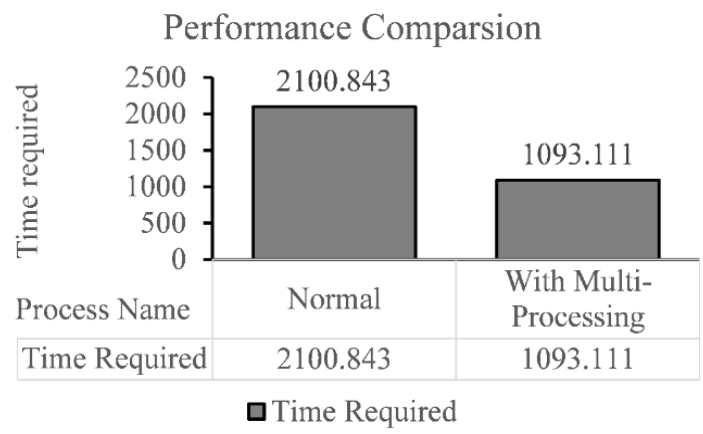

Fig. 7: Processing time comparison with and without parallelization.

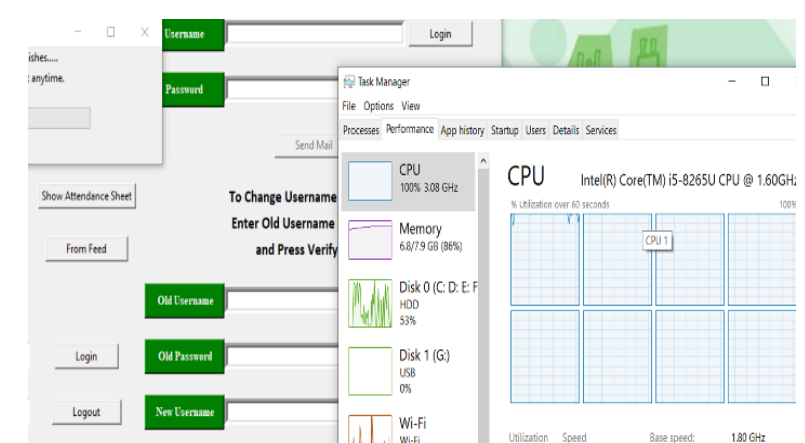

Fig. 8: CPU usage during the video processing on a 4 core 8 thread processor.

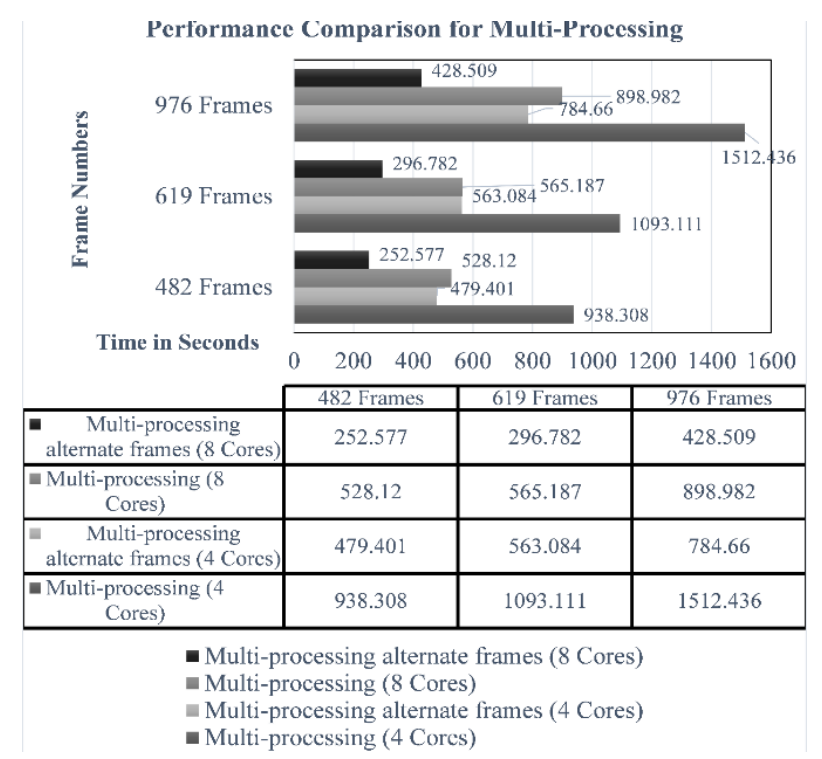

Fig. 9: Performance comparison of completion time required on two different machines with all frames and alternate frames processed. 
Trained model size is also another important comparison field. The larger the trained model size, the costlier is the hardware required. The size of the trained model for nine individual was found as close to 153 megabytes for the HAAR based system. Whereas, the trained model, which is basically the stored face encodings of the known people, in case of the second system (in a pickle file format) was only 9 kilobytes. Since the student number of the institute are bound to be more than nine individuals and the number of courses and classes are also an issue, the file size will increase dramatically. Another aspect of this works result evaluation is in terms of accuracy comparison. The system is compared to a HAAR cascade and LBPH based system trained using the images of the same students, but in much larger numbers (compared to this system which takes only one image for training) and with trained models much larger in size. The supplied image and the corresponding outputs based on HAAR Cascade-LBPH and HOG features, facial embedding and dlib model (ResNet based) are shown in Figure 10. It is obvious with the naked eye that differences are present in two results, for same input. These discrepancies are shown in Table 2.

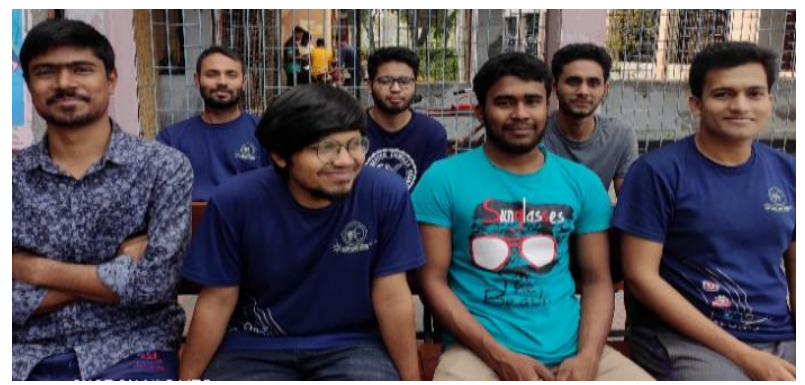

(a)

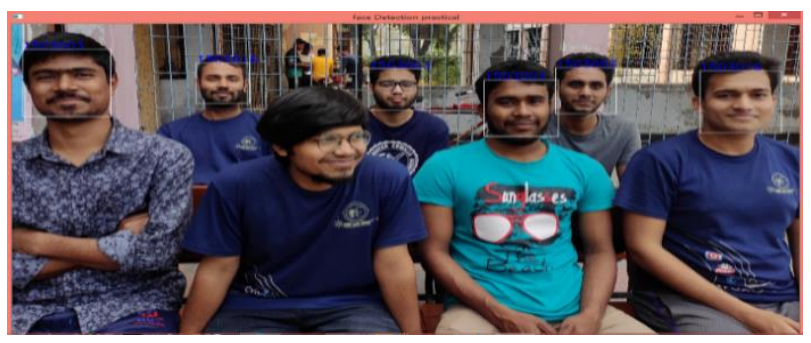

(b)

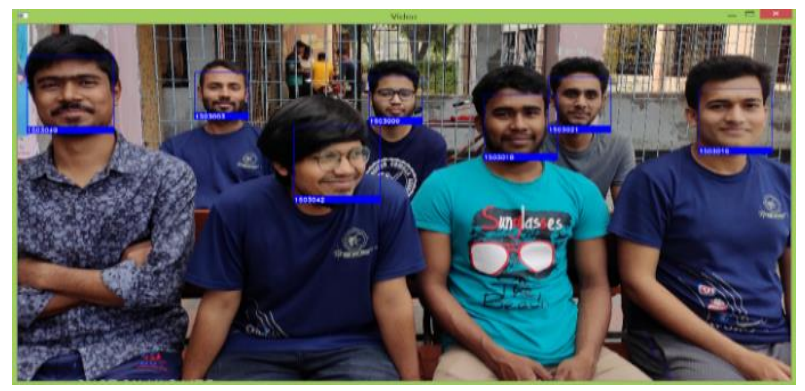

(c)
Fig.10: (a) Input image (b) Viola-Jones based HAAR Cascade and LBPH recognizer and (c) HOG and facial landmark, embedding, dlib model.

Table 2 . Difference in recognition and detection performance between the two systems.

\begin{tabular}{|c|c|c|c|c|c|}
\hline $\begin{array}{c}\text { Database } \\
\text { Image }\end{array}$ & $\begin{array}{c}\text { HAAR } \\
\text { based }\end{array}$ & $\begin{array}{l}\text { HOG } \\
\text { based }\end{array}$ & $\begin{array}{l}\text { Original } \\
\text { ID }\end{array}$ & $\begin{array}{c}\text { HAAR } \\
\text { ID }\end{array}$ & HOG ID \\
\hline & & & 1503003 & 1503016 & 1503003 \\
\hline & & & 1503009 & 1503003 & 1503009 \\
\hline & & & 1503018 & 1503003 & 1503018 \\
\hline & & & 1503021 & 1503003 & 1503021 \\
\hline & & & 1503042 & $\begin{array}{l}\text { Not } \\
\text { Detected }\end{array}$ & 1503042 \\
\hline & & & 1503049 & 1503003 & 1503049 \\
\hline
\end{tabular}

From Table 2, it is clear that for less training images the HOG and dlib based model performed better than the HAAR Cascade and LBPH based system.

These differences point to the system accuracy as well as the efficacy of the two methods for facial recognition. Requirement of training data is an important aspect of comparison as taking multiple images of the students can be cumbersome, even if it is extracted from a video of few seconds, introducing further complexities. For the purpose of testing, each of the students were asked to provide 30 facial images. The HAAR based system used 30 images per person for training the model whereas the second system required only one image per person and achieved far better result.

The system then successfully stored the attendance from each video footages to an initial excel sheet, from which it is updated to the attendance sheet. Figure 11 shows the initial attendance per frame recorded for two videos. Figure 12 shows the attendance sheet of a single teacher of a single course derived from the initial attendance sheet. The institute has two teachers per course. Figure 13 shows the individual and final attendant score sheets. Figure 14 shows the different 
email notifications to the students and course teachers receive from the system. All other activity such as generation of final attendance sheet, messaging to relevant personnel, checking individual percentages, admit card generation at the end of semester, end of semester, can be performed using the Graphical User Interface (GUI) designed for the system. Figure 15 shows the generated admit cards and Figure 16 shows the hardware architecture of the proposed system in simplified form.

\begin{tabular}{|c|c|c|c|c|}
\hline$\Delta$ & A & B & $\mathrm{C}$ & D \\
\hline 1 & Student Name & Roll & 315 & $g$ \\
\hline 2 & Md. Asoyat Hossain & 1503003 & 315 & $\mathrm{~g}$ \\
\hline 3 & K.M. Hayat & 1503008 & 0 & \\
\hline 4 & Rokunuzzaman Rakib & 1503009 & 315 & $\mathrm{~g}$ \\
\hline 5 & Abdullah Al Mamun Mozumdar & 1503016 & 315 & g \\
\hline 6 & Abul Bashar & 1503018 & 315 & 4 \\
\hline 7 & Md. Mostafizur Rahman & 1503021 & 315 & 9 \\
\hline 8 & Tanjim Ahsan Pial & 1503042 & 313 & 3 \\
\hline 9 & Al Amin Arif & 1503049 & 315 & 9 \\
\hline 10 & Nayeema Hasan & 1503060 & 0 & \\
\hline 11 & Azmol Ahmed Fuad & 1503011 & 0 & \\
\hline
\end{tabular}

Fig. 11: Initial attendance sheet.

\begin{tabular}{|c|c|c|c|c|c|c|c|}
\hline 2 & A & B & c & D & E & $\mathrm{F}$ & G \\
\hline 1 & Name & Roll & $02 / 15 / 20$ & $02 / 15 / 21$ & $102 / 16 / 20$ & $02 / 16 / 20$ & $02 / 16$ \\
\hline 2 & Md. Asoyat Hossain & 1503003 & 1 & & 1 & 1 & 1 \\
\hline 3 & K. M. Hayat & 1503008 & 1 & 1 & 1 & 1 & 1 \\
\hline 4 & Rokunuzzaman Rakib & 1503009 & c & 0 & 0 & 0 & 0 \\
\hline 5 & Abdullah Al Mamun Mojumdar & 1503016 & 1 & & 1 & 1 & 1 \\
\hline 6 & Abul Bashar & 1503018 & c & & 0 & 0 & 0 \\
\hline 7 & Md. Mostafizur Rahman & 1503021 & 1 & 1 & 1 & 1 & 1 \\
\hline 8 & Tanjim Ahsan Pial & 1503042 & c & 0 & 0 & 0 & 0 \\
\hline 9 & Al Amin Arif & 1503049 & c & & 0 & 0 & 0 \\
\hline 10 & Nayeema Hassan & 1503060 & 1 & 0 & 0 & 1 & 0 \\
\hline 11 & Azmol Ahmed Fuad & 1503011 & 1 & 1 & 1 & 1 & 1 \\
\hline
\end{tabular}

Fig. 12: Attendance sheet of a teacher of a course.

\begin{tabular}{|c|c|c|c|c|c|}
\hline 4 & A & B & C & D & E \\
\hline 1 & Name & Roll & Percent Attendance & Total Classes & Marks \\
\hline 2 & Md. Asoyat Hossain & 1503003 & 83.33 & 42 & 25 \\
\hline 3 & K. M. Hayat & 1503008 & 55.95 & & 16.79 \\
\hline 4 & Rokunuzzaman Rakib & 1503009 & 28.57 & & 8.57 \\
\hline 5 & Abdullah Al Mamun Mojumdar & 1503016 & 44.05 & & 13.21 \\
\hline 6 & Abul Bashar & 1503018 & 9.52 & & 2.86 \\
\hline 7 & Md. Mostafizur Rahman & 1503021 & 54.76 & & 16.43 \\
\hline 8 & Tanjim Ahsan Pial & 1503042 & 16.67 & & 5 \\
\hline 9 & Al Amin Arif & 1503049 & 23.81 & & 7.14 \\
\hline 10 & Nayeema Hassan & 1503060 & 42.86 & & 12.86 \\
\hline 11 | & omol $\Delta$ hmed Fular & 1502011 & fa $>0$ & & 1920 \\
\hline
\end{tabular}

(a)

\begin{tabular}{|c|c|c|c|c|c|}
\hline 4 & A & B & c & D & $\mathrm{E}$ \\
\hline 1 & Name & Roll & Percent Attendance & Total Classes & Marks \\
\hline 2 & Md. Asoyat Hossain & 1503003 & 80 & 55 & 24 \\
\hline 3 & K. M. Hayat & 1503008 & 42.73 & & 12.82 \\
\hline 4 & Rokunuzzaman Rakib & 1503009 & 38.18 & & 11.45 \\
\hline 5 & Abdullah Al Mamun Mojumdar & 1503016 & 48.18 & & 14.45 \\
\hline 6 & Abul Bashar & 1503018 & 20 & & 6 \\
\hline 7 & Md. Mostafizur Rahman & 1503021 & 58.18 & & 17.45 \\
\hline 8 & Tanjim Ahsan Pial & 1503042 & 27.27 & & 8.18 \\
\hline 9 & Al Amin Arif & 1503049 & 34.55 & & 10.37 \\
\hline 10 & Nayeema Hassan & 1503060 & 32.73 & & 9.82 \\
\hline 11 & Azmol Ahmed Fuad & 1503011 & 50.91 & & 15.27 \\
\hline
\end{tabular}

(b)

\begin{tabular}{|c|c|c|c|c|c|}
\hline 4 & A & B & C & D & $E$ \\
\hline 1 & Name & Roll & Percent Attendance & Total Classes & Marks \\
\hline 2 & Md. Asoyat Hossain & 1503003 & 83.33 & 42 & 25 \\
\hline 3 & K. M. Hayat & 1503008 & 55.95 & & 16.79 \\
\hline 4 & Rokunuzzaman Rakib & 1503009 & 28.57 & & 8.57 \\
\hline 5 & Abdullah Al Mamun Mojumdar & 1503016 & 44.05 & & 13.21 \\
\hline 6 & Abul Bashar & 1503018 & 9.52 & & 2.86 \\
\hline 7 & Md. Mostafizur Rahman & 1503021 & 54.76 & & 16.43 \\
\hline 8 & Tanjim Ahsan Pial & 1503042 & 16.67 & & 5 \\
\hline 9 & Al Amin Arif & 1503049 & 23.81 & & 7.14 \\
\hline 10 & Nayeema Hassan & 1503060 & 42.86 & & 12.86 \\
\hline 11 & Armol $\Delta h m e d$ Filad & 15ก२ก१11 & 64 20 & & 1920 \\
\hline
\end{tabular}

(c)

Fig. 13: (a), (b) Evaluation records from two different teachers of the same course, and (c) final evaluation record in the system.

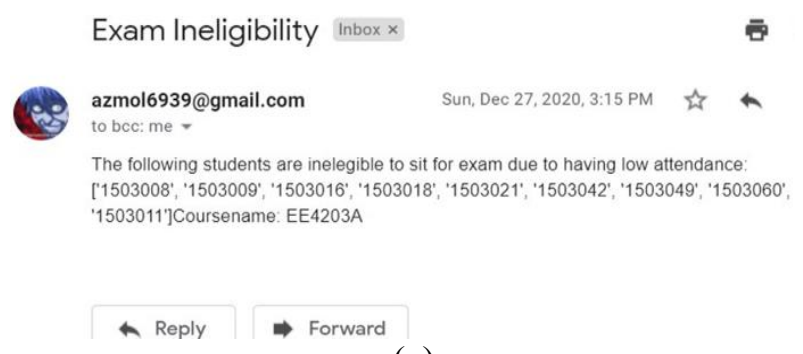

(a)

Attendance is too low Inbox $x$

azmol6939@gmail.com

to bcc: me -

Your attendance percentage is 54.18. Contact your teacher in EE4203A

$$
\rightarrow \text { Reply } \Rightarrow \text { Forward }
$$

(b)

Attendance is too low Inbox $x$

azmol6939@gmail.com

to bcc: me -

Your attendance percentage is 54.18. Contact your teacher in EE4203A

$$
\text { Reply } \Rightarrow \text { Forward }
$$

(c)

Fig.14: Email notification (a) to the course teacher with the list of students below required attendance in relevant course, (b) to the student with low percentage of attendance with course number and (c) from the teacher with course number for contact purposes.

The implemented system uses a traditional flat row and column-based classroom arrangement. It is clearly evident that such a setup would not be able to record attendance in a class full of students. Even alternating seating arrangement (every other seat in each row and column are left empty) would not be 
able to cover the whole class from the front. Thus, the ideal arrangement for this system would be gallery style seating arrangement (with enough height differences between each row) and an array of cameras focusing on certain portions of the classroom constantly. This would help to capture the faces of all the students without informing them. The entire rows and columns will be utilized. This would also eliminate the necessity for alternating seating arrangement to avoid facial obstruction.

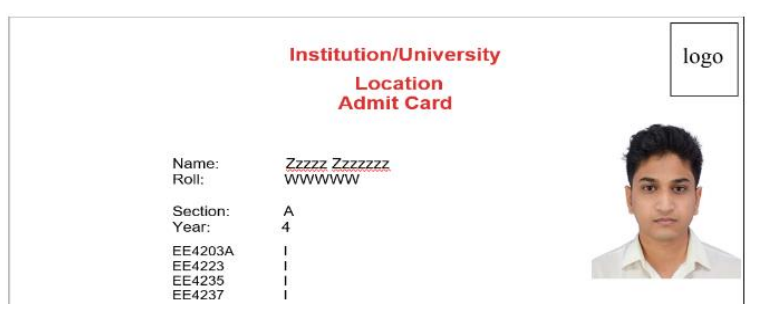

(a)

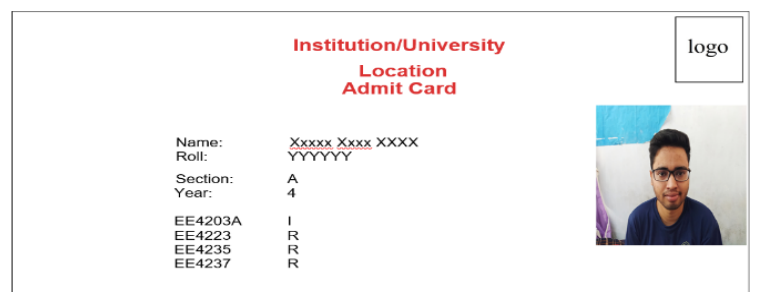

(b)

Fig. 15: Admit card (a) ineligible for all courses and (b) ineligible for one of the courses.

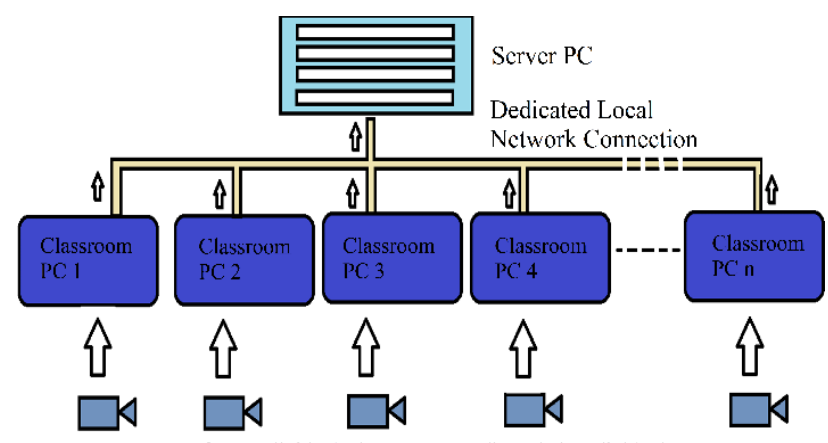

Cameras from Individual Classrooms Feeding Their Individual Systems

Fig. 16: Hardware architecture of the proposed system in simplified manner.

Figure 17 shows the ideal seating arrangement for the designed system. The records are updated on the local machine and the central server to be made available to other parties. The trained model and training data too is kept on the main server and only a copy of the trained model, alongside the attendance sheets are kept on the local machine, offering redundancy.

\section{Graphical User Interface (GUI)}

The implemented system has been incorporated in two GUI applications with installer files, for distribution. The client-side GUI is segmented into different blocks for different users. Each block user has their own login passwords. The blocks with the most features have the most number of buttons. The "Teachers Block" and the "Administration Block" serving the most key functions have the most features.

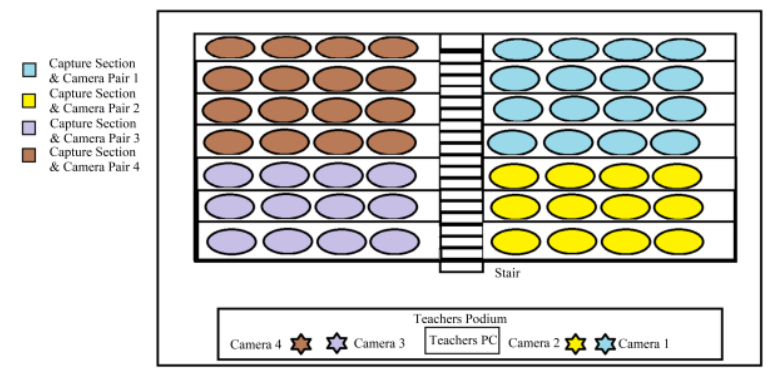

(a)

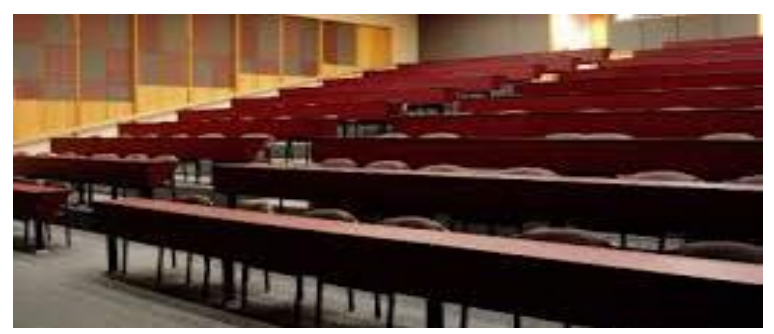

(b)

Fig. 17: (a) The proposed seating and hardware arrangement for the proposed system, where the same colour pair denote the camera and seat group and (b) gallery or stadium type seating arrangement in real life.

The attendance recording, uploading to the main server, calculating the percentage and marks, checking percentage and finally mailing the relevant personnel can be controlled by the "Teachers Block". The model training, device configuration and server operation is done by the administrative personnel, using the "Administration Block" features of the graphical user interface (GUI). The other blocks serve purposes like creating final mark sheet, mailing related course teachers, admit card generation etc. Figure 18 shows the two GUIs for the client and server side. Figure 19 shows various methods of recording attendance from the GUI. It is worth noting that the system was successfully able to identify the individual even with slight changes to the face (i.e., grown beard) against the original training image supplied. 


\section{Conclusion}

This work takes an attempt to design an efficient system for attendance documentation and supervision using facial detection and recognition techniques. The performance in recognizing slightly side-faced faces and natural facial changes (hair and beard growth) has also been evaluated. The training data and trained data size has been compared with another system in order to establish this systems supremacy.

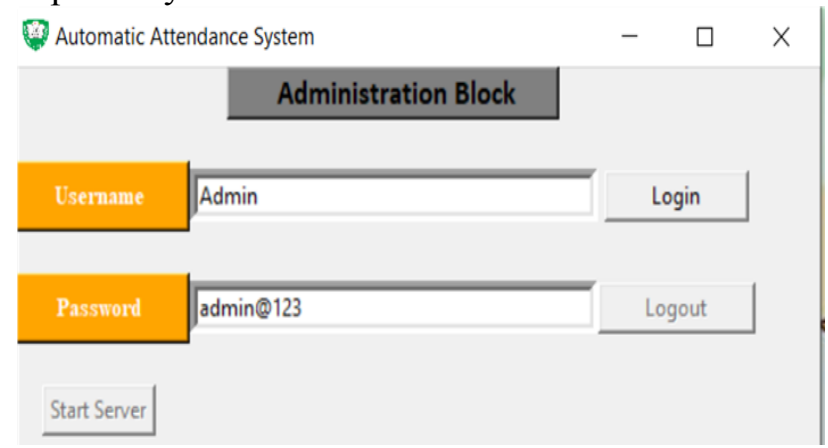

(a)

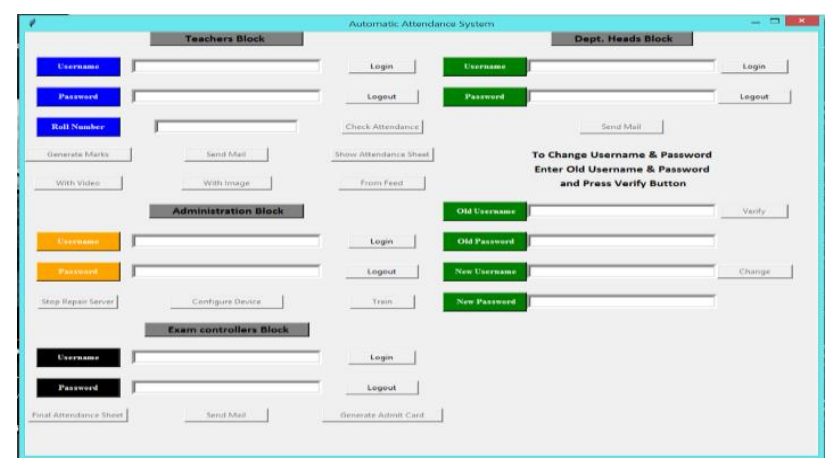

(b)

Fig. 18: GUI for (a) Administrative use of server control, and (b) Client-side users.

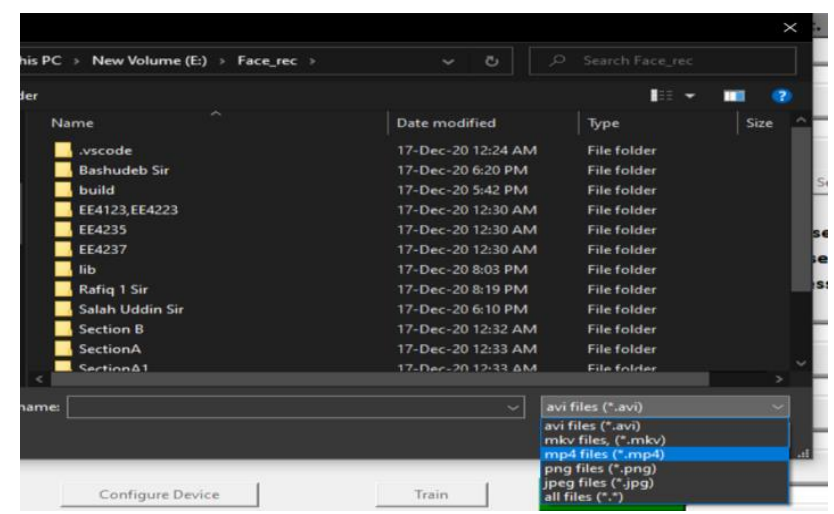

(a)

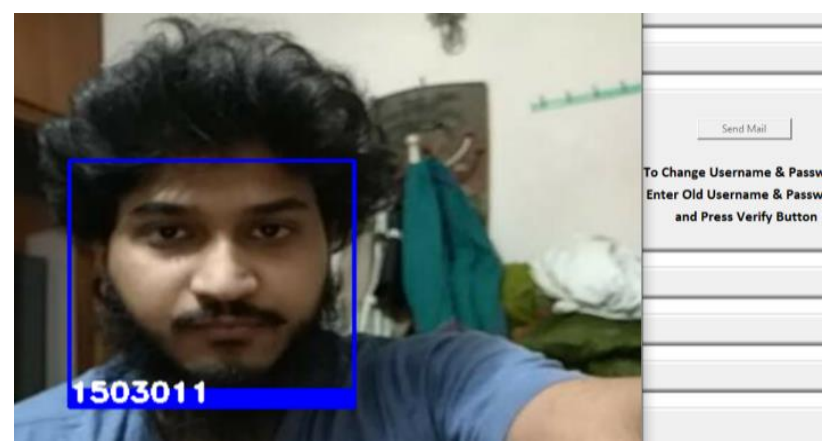

(b)

Fig. 19: Attendance recording via the "Teachers Block" of the GUI using (a) video file or image (/s) and (b) real time streaming.

(output of the processed stream shown).

The feasibility of its implementation in real life scenarios has also been evaluated in terms of training data requirement and processing speed. The work also showcases multiprocessing by CPU as a mean for faster and comparatively cheaper facial recognition. The model was found to have outperformed compared to the existing models. The findings of the model yield a positive implication for the model to expand its realms into areas with similar objectives.

\section{References:}

[1] A. Crawls, "Animetrics," 13 July 2008. Available:http://www.animetrics.com/Technol ogy/FRapplications.html. [Accessed 29 February 2020].

[2] Z. Jian, Y. Ke, H. Zhen-yu and X. Yong, "A Collaborative Linear Discriminative Representation Classification Method for Face Recognition.," in International Conference on Artificial Intelligence and Software Engineering, Lancaster, PA, 2014.

[3] J. Lei, T. Lay, C. Weiland and C. Lu, "A Combination of Spatiotemporal ICA and Euclidean Features for Face Recognition," Artificial Intelligence in Theory and Practice, vol. 217, no. 2006, pp. 395-403, 2006.

[4] P. Viola and M. Jones, "Robust Real-time Object Detection," in Second International Workshop on Statistical and Computational Theories, Vancouver, canada, 2001.

[5] E.T. Chin, W. J. Chew, and F. Choong, "Automated Attendance capture and Tracking System," Journal of Engineering Science and Technology, no. EURECA 2014 Special Issue, pp. 45-59, 2015.

[6] A. Raghuwanshi and P. Swami, "An Automated Classroom Attendance System," in $2^{\text {nd }}$ 
IEEE International Conference on Recent Trends in Electronics Information \& Communication Technology, Bangalore, 2017.

[7] N. D. Veer and B. F. Momin, "An automated attendance system using video," in IEEE International Conference on Recent Trends In Electronics Information Communication Technology, Bangalore, 2016.

[8] B. Surekha, K. J. Nazare, S. V. Raju and N. Dey, "Attendance Recording System Using Partial face Recognition Algorithm," Studies in Computational Intelligence, vol. Intelligent Techniques in Signal Processing, no. 2017, pp. 293-319, 2016.

[9] A. Patil and M. Shukla, "Implementation of Classromom Attendance System Based on Face Recognition in Class," International Journal of Advances in Engineering \& Technology, vol. 7, no. 3, pp. 974-979, 2014.

[10] M. Fuzail, H. Nouman, M. Mushtaque, B. Raza, A. Tayyab and M. Talib, "Face Detection System for Attendance of Class' Students," International Journal of Multidisciplinary Sciences and Engineering, vol. 5, no. 4, pp. 6-10, 2014.

[11] A. Geitgey, "Medium," 13 July 2016. [Online]. Available: https://medium.com/ @ageitgey/machine-learning-is-fun-part-3deep-learning-and-convolutional-neuralnetworks- [Accessed 28 February 2020].

[12] K. Rohini, S. Sanagala, V. Rathnam and C. R. Babu, "Face Recogntion Based Attendance System for CMR College of Engineering and Technology," International Journal of Innovative Technology \& Engineering,vol. 8, no. 452, pp. 127-129, 2019.

[13] A. Jha, "Class Room Attendance System using Facial Recognition System," International Journal of Mathematics, Science, Technology and Management, vol. 2, no. 3, pp. 4-7, 2007.

[14] N. K. Balcoh, M. Yousaf, W. Ahmad and M. Baig, "Algorithm for Efficient Attendance Management: Face Recognition based approach," International Journal of Computer Science, vol. 9, no. 4, pp. 146-150, 2012.

[15] V. Shehu and A. Dika, "Using Real Time Computer Vision Algorithms in Automatic Attendance Management Systems," in proceeding of the $32^{\text {nd }}$ International Conference on Information Technology Interfaces, Cavtat, 2010.

[16] D. Sunaryono, J. Siswantoro and R. Anggoro, "An Android Based Course Attendance System using Face Recognition," Journal of
King Saud University-Computer and Information Sciences, vol. 33, no. 3, pp 304312, 2019.

[17] A. Waingankar, A. Upadhyay, R. Shah, N. Pooniwala and P. Kasambe, "Face Recognition based Attendance Management System using Machine Learning," International Research Journal of Engineering and Technology (IRJET), vol. 5, no. 6, pp. 1979-1985, 2010.

[18] A. Geitgey, "Medium," 24 July 2016. [Online]. Available: https://medium.com/@ageitegy/machinelearning-is-fun-part-4-modern-facerecognition-with-deep-learning[Accessed 29 February 2020].

[19] N. Dalal and B. Triggs, "Histograms of Oriented Gradients for Human Detection," in $5^{\text {th }}$ IEEE Computer Society Conference on Computer Vision and Pattern Recognition, San Diego, CA, USA, 2005.

[20] V. Kazemi and J. Sullivan, "One Millisecond Face Alignment with an Ensemble of Regression Trees," in Proceedings of the IEEE Conference on Computer Vision and Pattern Recognition, Long Beach, 2014.

[21] D. Wang, R. Fu and Z. Luo, "Classroom Attendance Auto-management Based on Deep Learning," in of 2nd International Conference on Education, Sports, Arts and Management Engineering, Zhengzhou, 2017.

[22] https://github.com/seetaface/SeetaFaceEngine

[23] F. Schroff, D. Kalenichenko and J. Philbin, "FaceNet: A Unified Embedding for Face Recognition and Clustering," in Proceedings of the IEEE Conference on Computer Vision and Pattern Recognition, Boston, MA, USA, 2015.

[24] D. KIng, "Dlib c++ Library," 12 February 2017. [Online]. Available: http://blog.dlib.net/2017/02/high-quality-facerecognition-with-deep.html. [Accessed 27 February 2020].

\section{Contribution of Individual Authors to the Creation of a Scientific Article (Ghostwriting Policy)}

Md. Salah Uddin Yusuf has carried out the supervision, Conceptualization, Manuscript writing, editing and review.

Azmol Ahmed Fuad has carried out the simulation, experimental analysis and optimization, writing.

All authors have read and agreed to the published version of the manuscript. 


\section{Sources of Funding for Research Presented} in a Scientific Article or Scientific Article Itself

This research did not receive any specific grant from funding agencies in the public, commercial, or nonprofit sectors.

\section{Creative Commons Attribution License 4.0}

\section{(Attribution 4.0 International, CC BY 4.0)}

This article is published under the terms of the Creative Commons Attribution License 4.0

https://creativecommons.org/licenses/by/4.0/deed.en US 